Research Paper

\title{
Risk Prediction for Early Mortality in Patients with Newly Diagnosed Primary CNS Lymphoma
}

\author{
Chia-Hsin Lin ${ }^{1,2}$, Ching-Fen Yang ${ }^{3}$, Huai-Che Yang ${ }^{4,5}, \mathrm{Li}_{-} \mathrm{Yu} \mathrm{Fay}^{4}$, Chiu-Mei Yeh ${ }^{6,7}$, Ai-Seon Kuan ${ }^{8}$, \\ Hao-Yuan Wang2,6, Jyh-Pyng Gau 2,6, Liang-Tsai Hsiao 2,6, Tzeon-Jye Chiou', ${ }^{2,}$, Po-Min Chen 2,6, Yao-Chung \\ $\mathrm{Liu}^{2,6}$, Po-Shen Ko ${ }^{2,6}$, Jin-Hwang Liu'6,10,11, Chia-Jen Liu6,7凶 \\ 1. Department of Radiation Oncology, Linkou Chang Gung Memorial Hospital Medical Center, Taoyuan City, Taiwan \\ 2. School of Medicine, National Yang-Ming University, Taipei, Taiwan \\ 3. Department of Pathology and Laboratory Medicine, Taipei Veterans General Hospital, Taipei, Taiwan \\ 4. Department of Neurosurgery, Taipei Veterans General Hospital \\ 5. Institute of Brain Science, National Yang-Ming University \\ 6. Division of Hematology and Oncology, Department of Medicine, Taipei Veterans General Hospital, Taipei, Taiwan \\ 7. Institute of Public Health, National Yang-Ming University, Taipei, Taiwan \\ 8. Cancer Epidemiology Unit, Nuffield Department of Population Health, University of Oxford, Oxford, OX3 7LF, UK \\ 9. Division of Transfusion Medicine, Taipei Veterans General Hospital, Taipei, Taiwan \\ 10. Institute of Biopharmaceutical Sciences, National Yang-Ming University, Taipei, Taiwan \\ 11. Chong Hin Loon Memorial Cancer and Biotherapy Research Center, National Yang-Ming University, Taipei, Taiwan \\ $\triangle$ Corresponding author: Dr. Chia-Jen Liu, Division of Hematology and Oncology, Department of Medicine, Taipei Veterans General Hospital. No. 201 Shipai \\ Road, Sec. 2, Taipei 112, Taiwan. Email: chiajenliu@gmail.com
}

(C) The author(s). This is an open access article distributed under the terms of the Creative Commons Attribution License (https://creativecommons.org/licenses/by/4.0/). See http://ivyspring.com/terms for full terms and conditions.

Received: 2018.12.21; Accepted: 2019.05.21; Published: 2019.07.05

\begin{abstract}
Background: Overall survival of patients with primary CNS lymphoma (PCNSL) has improved since the introduction of immunochemotherapy. However, up to 10-15\% of PCNSL patients still die shortly after diagnosis. In the present study, we aimed to investigate the risk factors of early mortality (death within 60 days after diagnosis) in patients with PCNSL.
\end{abstract}

Methods: We included newly diagnosed PCNSL patients in a tertiary medical center in Taiwan between January 1, 2002 and May 31, 2018. Clinical risk factors were collected and compared between PCNSL patients who had and did not have early mortality.

Results: A total of 133 consecutive patients with PCNSL were included in this study. Approximately $9.8 \%$ of the PCNSL patients had early mortality. In multivariate analysis, age $\geq 80$ (adjusted hazard ratio [HR] 3.34, 95\% confidence interval $[\mathrm{Cl}] 1.01-11.04, p=0.048$ ) and involvement of the basal ganglia (adjusted $\mathrm{HR} 4.85,95 \% \mathrm{Cl}$ 1.47-15.95, $p=0.009$ ) were identified as independent risk factors of early mortality. Use of MTX-based chemotherapy served as an independent protective factor for early mortality (adjusted $\mathrm{HR} 0.19,95 \% \mathrm{Cl}$ $0.05-0.67, p=0.010$ ). Infection and tumor-associated mass effect contributed most to early mortality.

Conclusion: Early mortality is not uncommon in patients with PCNSL. Identification of patients with higher risk may help clinicians with initiating appropriate surveillance and management.

Key words: Early mortality, epidemiology, primary CNS lymphoma, prognostic factors

\section{Introduction}

Primary central nervous system (CNS) lymphoma (PCNSL) is a relative rare disease, accounting for approximately $4 \%$ of all primary CNS tumors [1-3]. It is an extranodal variant of non-Hodgkin's lymphoma (NHL), which is confined to the brain, leptomeninges, spinal cord and eyes, and it is without systemic involvement $[4,5]$. Recently, a steady increase in incidence of PCNSL was reported, especially in elderly patients ( $\geq 65$ years), which represents the majority of immunocompetent PCNSL $[6,7]$.

When compared with systemic diffuse large B-cell lymphoma (DLBCL), the prognosis of PCNSL is poor. PCNSL patients who do receive no treatment have a rapidly fatal course, with a median survival of 3.3 months [8]. The prognosis of PCNSL patients, 
however, has substantially improved in the recent two decades due to the introduction of induction immunochemotherapy (rituximab, methotrexate, etc.) in combination with autologous hematopoietic stem cell transplantation (auto-HSCT) as consolidation for eligible patients [3, 9]. Omuro et al. reported remarkable results in a phase-2 study for patients of newly diagnosed PCNSL with a three-year overall survival (OS) rate and progression-free survival (PFS) of up to $81 \%$ and $79 \%$, respectively [10]. Nevertheless, despite the advancement of novel therapies and improvement of OS, some patients still saw early mortality [10]. Furthermore, patients with significant comorbidities or critical conditions were usually excluded from clinical trials, making the results not generalizable to all PCNSL patients [11-14].

To date, the International Extranodal Lymphoma Study Group (IELSG) score, Memorial Sloan Kettering Cancer Center (MSKCC) score, and the Nottingham-Barcelona (NB) model make up three major prognostic scoring systems [15, 16]. Some parameters-for example, age, performance status (PS), serum lactate dehydrogenase (LDH) level, cerebrospinal fluid (CSF) total protein concentration, tumor localization (deep vs. superficial) and number of lesions - are well-established prognostic factors in the aforementioned models [15-17]. However, the current scoring systems for PCNSL are used for prediction of OS. Hervé et al. further demonstrated that the prognostic values of some of these parameters may vary from time to time [18]. Given that PCNSL is a heterogeneous disease, and the survival duration ranges from a month to more than five years, $[15,16]$ the present scoring systems may not extrapolate to accurately predict the outcome of PCNSL patients during a particular period after diagnosis.[18]

However, few studies have delineated the incidence or risk factors related specifically to early mortality in patients with PCNSL. In addition, patients who had early mortality were not studied in randomized control trials, making the evidence from observational studies very important. Accordingly, we conducted a longitudinal observational study to address this issue.

\section{Patients and Methods}

\section{Study Population}

This study consecutively enrolled patients who were newly diagnosed with PCNSL between January 1, 2002 and May 31, 2018 at Taipei Veterans General Hospital. Patients were eligible if they had non-Hodgkin's lymphoma exclusively involving the CNS, leptomeninges, cranial nerves, or eyes. Diagnosis was made on stereotactic or surgical biopsy, CSF cytology analysis, or vitreous biopsy and was reviewed by at least one hematologist and one pathologist. Patients who had suspected PCNSL lesions demonstrated only by brain magnetic resonance image (MRI) or computed tomography (CT) but without histological confirmation were not included. Exclusion criteria were human immunodeficiency virus seropositivity, other immunodeficiency diseases or evidence of systemic NHL, as revealed by CT images of the chest, abdomen, pelvis and bone marrow aspiration and biopsy. Follow-up was continued until death, dropout, 60 days, or October 10, 2018. The Institutional Review Board of Taipei Veterans General Hospital issued a formal written waiver for the need for consent (no. 2016-05-003BC).

\section{Data Collection}

Medical records for all PCNSL patients were reviewed. Clinical characteristics and laboratory data at diagnosis, such as age, sex, date of diagnosis, date of death, cause of death, comorbidities, treatment, chemotherapy regimen, LDH levels [15], hemoglobin levels, white blood cell (WBC) count, platelet count and albumin level were retrieved. Patients' serum $\beta 2$-microglobulin level was measured by immunoassay (upper limit of normal, $1.61 \mathrm{mg} / \mathrm{l}$ ). Cutoff values of hemoglobin, albumin, WBC counts, and platelet counts were $8.5 \mathrm{~g} / \mathrm{dl}, 3 \mathrm{~g} / \mathrm{dl}, 10,000 / \mu \mathrm{l}$, and $100,000 / \mu \mathrm{l}$, respectively, which are correlated with the early mortality seen in systemic NHL in previous studies [19, 20]. Performance status is defined according to Eastern Cooperative Oncology Group (ECOG) criteria [15, 21, 22]. There is no formal definition of early mortality; for the present study, we chose 60 days from disease diagnosis, according to studies of other hematologic malignancies [23, 24].

\section{Statistical Analysis}

Patients' demographic and clinical characteristics with and without early mortality are presented as the total number $(n)$ and proportion (\%). Data are presented as medians and interquartile ranges (IQR) for skewed data. In the survival analysis, hazard ratios (HRs) and the $95 \%$ confidence intervals (CIs) were calculated using Cox proportional hazards models. We used multivariate analysis to calculate adjusted HRs while adjusting for possible confounding factors, including the demographic and clinical characteristics of patients. All risk factors with $p<0.1$ in the univariate model were selected for stepwise-selection Cox proportional hazards model. To deal with immortal time bias, we analyzed treatment as time-dependent covariates in the Cox proportional hazards model. Treatments included 
radiotherapy and chemotherapy agents, such as methotrexate (MTX), vincristine, cyclophosphamide, cytarabine, rituximab, and corticosteroid. Data management and all statistical analysis was performed using SAS 9.4 software (SAS Institute Inc., Cary, NC). All statistically significant levels were set at $p<0.05$.

\section{Results}

\section{Clinical Characteristics of the Study Population}

A total of 179 patients with CNS lymphoma diagnosed at Taipei Veterans General Hospital between January 1, 2002 and May 31, 2018 were identified. After reviewing the pathology and clinical data, patients who were misclassified into PCNSL $(n=$ $31)$, diagnosed with secondary CNS lymphoma $(n=9)$ or with HIV infection $(n=6)$ were excluded. Finally, 133 PCNSL patients were enrolled in the study
(Figure 1). Table 1 summarizes the clinical characteristics of PCNSL patients. The median age was 64 years (range 22-88 years), and $56.4 \%$ were male. Sixty-eight patients (51.1\%) had an ECOG PS more than 1 . The most common sites were frontal $(38.4 \%)$ and basal $(36.8 \%)$ lobe. The pathological diagnosis of all patients was diffuse large B-cell lymphoma. The details of treatment regimens are shown in Figure 2. Of the 133 PCNSL patients, 118 $(88.7 \%)$ received treatment after initial diagnosis, and MTX was the most commonly used drug $(n=104)$ in the frontline treatment, followed by rituximab $(n=$ $76)$, high-dose cytarabine $(n=30)$, and vincristine $(n=$ 22). Radiotherapy was administered in 67 patients (50.4\%). Radiotherapy was administered to the whole brain with a median dose (IQR) of $30(24-40)$ Gy, followed or not by a tumor-bed boost with 41 (36-46) Gy.

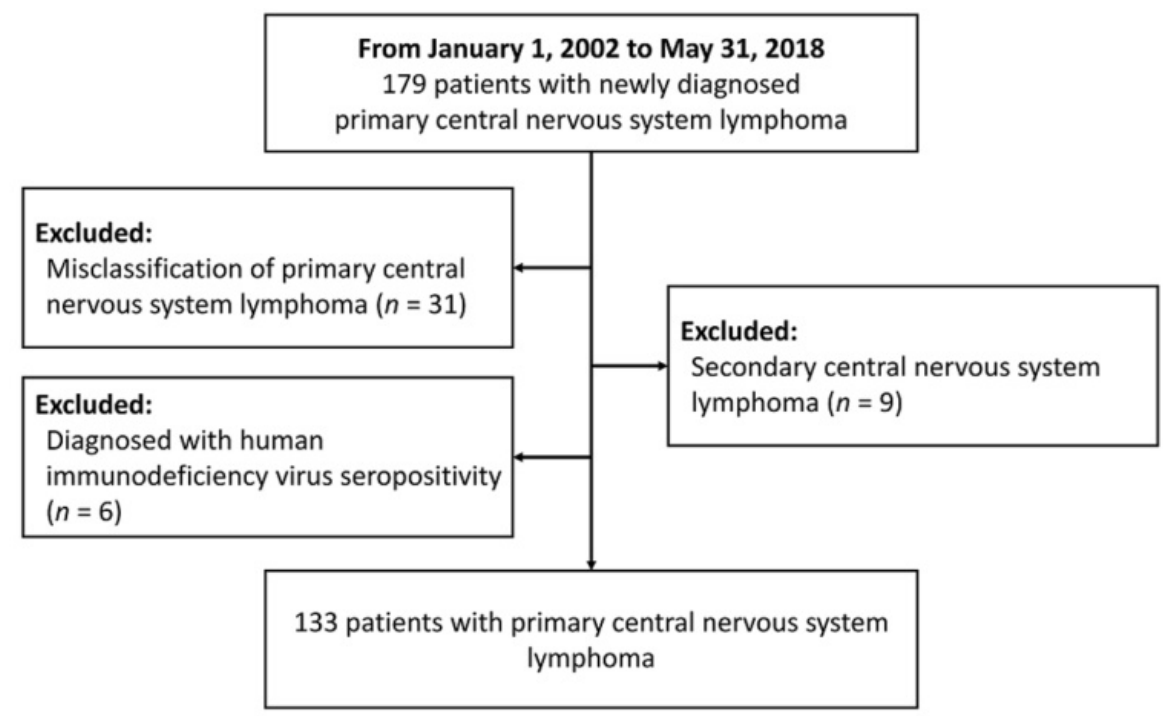

Figure 1. The flow chart for this study

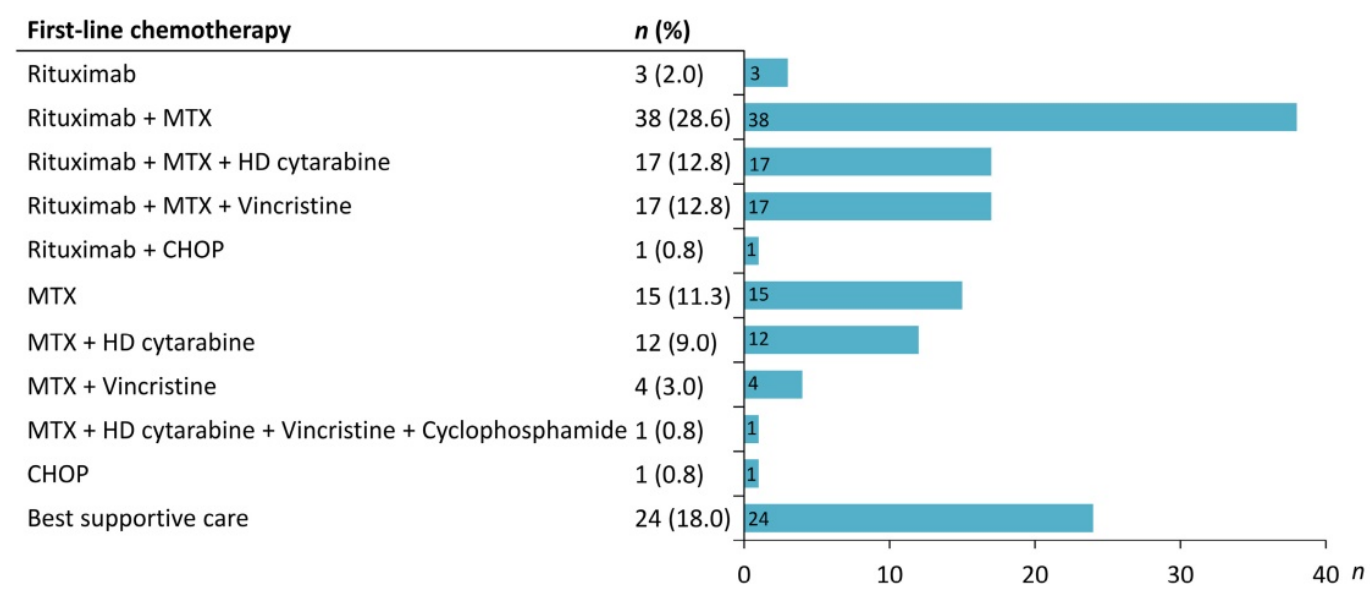

Figure 2. Treatment in the 133 primary CNS lymphoma patients studied. MTX, methotrexate; HD, high dose; CHOP, cyclophosphamide, doxorubine, vincristine, prednisone. 
Table 1. Baseline PCNSL patient characteristics

\begin{tabular}{|c|c|c|c|}
\hline \multirow[t]{3}{*}{ Characteristics } & Total $^{*}$ & $\begin{array}{l}\text { Early mortality } \\
\text { (60 days) }\end{array}$ & $\begin{array}{l}\text { Non-early } \\
\text { mortality }\end{array}$ \\
\hline & $n=133$ & $n=13$ & $n=120$ \\
\hline & $n(\%)$ & $n(\%)$ & $n(\%)$ \\
\hline $\begin{array}{l}\text { Median age, years } \\
\text { (range) }\end{array}$ & $64(22-88)$ & $70(44-87)$ & $64(22-88)$ \\
\hline$\geq 80$ & $16(12.0)$ & $4(30.8)$ & $12(10.0)$ \\
\hline$<80$ & $117(88.0)$ & $9(69.2)$ & $108(90.0)$ \\
\hline \multicolumn{4}{|l|}{ Sex } \\
\hline Male & $75(56.4)$ & $8(61.5)$ & $67(55.8)$ \\
\hline Female & $58(43.6)$ & $5(38.5)$ & $53(44.2)$ \\
\hline \multicolumn{4}{|l|}{ Site } \\
\hline Frontal lobe & $51(38.4)$ & $5(38.5)$ & $46(38.3)$ \\
\hline Parietal lobe & $37(27.8)$ & $3(23.1)$ & $34(28.3)$ \\
\hline Temporal lobe & $37(27.8)$ & $4(30.8)$ & $45(37.5)$ \\
\hline Occipital lobe & $23(17.3)$ & $4(30.8)$ & 21 (17.5) \\
\hline Basal ganglia & $49(36.8)$ & $9(69.2)$ & $40(33.3)$ \\
\hline Brain stem & $17(12.8)$ & $2(15.4)$ & 15 (12.5) \\
\hline Cerebellum & $18(13.5)$ & $3(23.1)$ & $15(12.5)$ \\
\hline Meninges & $1(0.8)$ & $0(0.0)$ & $15(12.5)$ \\
\hline Cranial nerves & $2(1.5)$ & $0(0.0)$ & $2(1.7)$ \\
\hline $\begin{array}{l}\text { Median max } \\
\text { diameter (IQR) }\end{array}$ & $3.4(2.6-4.5)$ & $4.0(3.2-4.3)$ & $3.3(2.6-4.5)$ \\
\hline \multicolumn{4}{|l|}{ Number of lesions } \\
\hline 1 & $66(49.6)$ & $8(61.5)$ & $58(48.3)$ \\
\hline 2 & $27(20.3)$ & $2(15.4)$ & $25(20.8)$ \\
\hline 3 & $17(12.8)$ & $0(0.0)$ & 17 (14.2) \\
\hline 4 & $8(6.0)$ & $0(0.0)$ & $8(6.7)$ \\
\hline$\geq 5$ & 15 (11.3) & $3(23.1)$ & $12(10.0)$ \\
\hline Deep brain lesions & $91(68.4)$ & $11(84.6)$ & $80(66.7)$ \\
\hline Midline shift & 46/131 (35.1) & 6/13 (46.2) & $40 / 118(33.9)$ \\
\hline \multicolumn{4}{|l|}{$\begin{array}{l}\text { Initial surgical } \\
\text { management }\end{array}$} \\
\hline Stereotactic biopsy & 85 (63.9) & $9(69.2)$ & $76(63.3)$ \\
\hline Open biopsy & $6(4.5)$ & $0(0.0)$ & $6(5.0)$ \\
\hline Partial resection & 42 (31.6) & $4(30.8)$ & $38(31.7)$ \\
\hline CSF involvement & $5 / 74(6.8)$ & $0 / 2(0.0)$ & $5 / 72(6.9)$ \\
\hline $\begin{array}{l}\text { Intraocular } \\
\text { involvement }\end{array}$ & $15 / 63(23.8)$ & $0 / 4(0.0)$ & $15 / 59(25.4)$ \\
\hline \multicolumn{4}{|l|}{ ECOG } \\
\hline $0-1$ & $65(48.9)$ & $2(15.4)$ & $63(52.5)$ \\
\hline$\geq 2$ & $68(51.1)$ & $11(84.6)$ & $57(47.5)$ \\
\hline \multicolumn{4}{|l|}{$\begin{array}{l}\text { Lab data, median } \\
\text { (IQR) }\end{array}$} \\
\hline $\begin{array}{l}\text { White blood cell, } \\
\text { /ul }\end{array}$ & $\begin{array}{l}9,700 \\
(7,200-13,200)\end{array}$ & $\begin{array}{l}10,400 \\
(6,700-13,600)\end{array}$ & $\begin{array}{l}9,650 \\
(7,200-13,150)\end{array}$ \\
\hline Hemoglobin, g/dl & $12.7(11.4-14.1)$ & $12.0(10.6-12.7)$ & $12.9(11.7-14.1)$ \\
\hline Platelet, /ul & $\begin{array}{l}199,000 \\
(157,000-270,000)\end{array}$ & $\begin{array}{l}171,000 \\
(143,000-288,000)\end{array}$ & $\begin{array}{l}200,500 \\
(158,000-268,500)\end{array}$ \\
\hline Albumin, g/dl & $3.7(3.2-4.1)$ & $3.5(3.0-3.8)$ & $3.8(3.3-4.1)$ \\
\hline $\begin{array}{l}\text { Lactate } \\
\text { dehydrogenase, } \\
\text { U/L }\end{array}$ & $\begin{array}{l}241.0 \\
(198.0-317.0)\end{array}$ & $\begin{array}{l}265.0 \\
(235.0-501.0)\end{array}$ & $\begin{array}{l}236.5 \\
(194.5-312.5)\end{array}$ \\
\hline CSF protein, mg/dl & $66.9(43.1-100.0)$ & $78.6(74.3-241.1)$ & $63.5(43.0-100.0)$ \\
\hline \multicolumn{4}{|l|}{ First-line treatments } \\
\hline MTX & $104(78.2)$ & $4(30.8)$ & $100(83.3)$ \\
\hline Rituximab & $76(57.1)$ & $2(15.4)$ & $74(61.7)$ \\
\hline Vincristine & $22(16.5)$ & $0(0.0)$ & $22(18.3)$ \\
\hline Cyclophosphamide & $1(0.8)$ & $0(0.0)$ & $1(0.8)$ \\
\hline Cytarabine & $30(22.6)$ & $0(0.0)$ & $30(25.0)$ \\
\hline Corticosteroid & $127(95.5)$ & $10(76.9)$ & $117(97.5)$ \\
\hline Radiotherapy & $67(50.4)$ & $2(15.4)$ & $65(54.2)$ \\
\hline
\end{tabular}

$\mathrm{IQR}$, interquartile range; BM, bone marrow; ECOG, Eastern Cooperative Oncology Group performance; MTX, methotrexate. * Including some missing value

\section{The Risk Factors of Early Mortality}

In the univariate analysis, we found that old age,
PCNSL with basal ganglia involvement, worse ECOG performance status, receiving MTX- and rituximab-based chemotherapy were significant predictors for early mortality in patients with PCNSL (Table 2). In the multivariate analysis, age $\geq 80$ (adjusted HR 3.34, 95\% CI 1.01-11.04, $p=0.048$ ) and PCNSL with basal ganglia involvement (adjusted HR 4.85, 95\% CI 1.47-15.95, $p=0.009$ ) remained statistically significant. Meanwhile, frontline MTX lowered the risk of early mortality (adjusted HR 0.19, 95\% CI 0.05-0.67, $p=0.01$ ) after adjustment with potential confounding factors. Whole brain radiation therapy (WBRT) and surgical partial resection, were not associated with early mortality in either univariate or multivariate analysis.

\section{Incidence and Causes of Early Mortality}

The median overall survival of the entire population was 8.4 years (IQR 2.5-not reach years). The sixty-day survival rate for PCNSL patients was $89.9 \%$ (95\% CI 83.2\%-94.0\%). Early mortality arose in 13 of the $133(9.8 \%)$ newly diagnosed PCNSL patients. The survival probability in PCNSL patients is shown in Figure 3. Mortality was related to PCNSL with mass effect in $5(38.5 \%)$, tumor bleeding in $1(7.7 \%)$ and disease progression in $1(7.7 \%)$ of early-mortality patients. Infections were also direct causes of mortality in $46.2 \%$ of early-mortality PCNSL patients $(n=6)$. Pneumonia directly caused death in four cases and sepsis attributed to two cases, accounting for $66.7 \%$ and $33.3 \%$ of the patients who died early of infection, respectively. The causes of early mortality are shown in Figure 4.

\section{Discussion}

Knowledge of risk factors and causes of deaths in PCNSL patients may help clinicians with identifying higher-risk patients and developing strategies for prevention of early mortality. However, clinical factors that affect early mortality have scarcely been studied and so far less investigated in clinical trials. To the best of our understanding, this is the largest study that examines risk factors of early mortality in PCNSL patients.

Our study reveals that old age (age $\geq 80$ ) and PCNSL with basal ganglia involvement were potential risk factors of early mortality (death within 60 days) in PCNSL patients. Our results are consistent with studies that reported factors predicting overall survival at a later stage $[15,25,26]$. This is also the first study that reports an association between MTX-based chemotherapy and reduced risk of early mortality in PCNSL patients. Furthermore, we report that infection and tumor-related mass effect are two major causes of early mortality among PCNSL patients. 
Table 2. Analysis of risk factors for mortality (60 days) in patients with PCNSL

\begin{tabular}{|c|c|c|c|c|}
\hline \multirow[t]{2}{*}{ Predictive variables } & \multicolumn{2}{|c|}{ Univariate analysis } & \multicolumn{2}{|l|}{$\begin{array}{l}\text { Multivariate } \\
\text { analysisa }\end{array}$} \\
\hline & HR (95\% CI) & $\begin{array}{l}P \\
\text { value }\end{array}$ & HR (95\% CI) & $\begin{array}{l}P \\
\text { value }\end{array}$ \\
\hline Age $\geq 80$ & $3.72(1.14-12.10)$ & 0.029 & $\begin{array}{l}3.34 \\
(1.01-11.04)\end{array}$ & 0.048 \\
\hline Sex (male) & $1.30(0.43-3.98)$ & 0.642 & & \\
\hline \multicolumn{5}{|l|}{ Site } \\
\hline Frontal lobe & $0.94(0.31-2.88)$ & 0.914 & & \\
\hline Parietal lobe & $0.78(0.22-2.84)$ & 0.709 & & \\
\hline Temporal lobe & $0.74(0.23-2.40)$ & 0.614 & & \\
\hline Occipital lobe & $0.93(0.21-4.20)$ & 0.926 & & \\
\hline Basal ganglia & $4.26(1.31-13.85)$ & 0.016 & $\begin{array}{l}4.85 \\
(1.47-15.95)\end{array}$ & 0.009 \\
\hline Brain stem & $1.36(0.30-6.14)$ & 0.688 & & \\
\hline Cerebellum & $2.05(0.57-7.47)$ & 0.274 & & \\
\hline Meninges & * & 0.995 & & \\
\hline Cranial nerves & * & 0.993 & & \\
\hline Max diameter $\geq 3.4$ & $2.18(0.67-7.08)$ & 0.195 & & \\
\hline Number of lesions $\geq 5$ & $2.58(0.71-9.37)$ & 0.150 & & \\
\hline Deep brain lesions & $2.70(0.60-12.20)$ & 0.196 & & \\
\hline Midline shift & $1.62(0.54-4.82)$ & 0.385 & & \\
\hline CSF involvement & * & 0.998 & & \\
\hline Intraocular involvement & * & 0.996 & & \\
\hline $\mathrm{ECOG} \geq 2$ & $5.48(1.21-24.70)$ & 0.027 & & \\
\hline \multicolumn{5}{|l|}{ Laboratory data } \\
\hline CSF protein $\geq 66.9 \mathrm{mg} / \mathrm{dl}$ & * & 0.995 & & \\
\hline $\mathrm{WBC} \geq 10,000 / \mathrm{ul}$ & $1.32(0.44-3.94)$ & 0.615 & & \\
\hline Hemoglobin $<8.5 \mathrm{~g} / \mathrm{dl}$ & * & 0.994 & & \\
\hline Platelets $\geq 100,000 / \mathrm{ul}$ & * & 0.993 & & \\
\hline $\mathrm{ANC} \geq 7,278 / \mathrm{ul}$ & $1.12(0.38-3.35)$ & 0.833 & & \\
\hline Albumin $<3 \mathrm{~g} / \mathrm{dl}$ & $1.85(0.40-8.59)$ & 0.430 & & \\
\hline $\begin{array}{l}\text { Lactate dehydrogenase } \geq 250 \\
\mathrm{U} / \mathrm{L}\end{array}$ & $1.57(0.48-5.16)$ & 0.454 & & \\
\hline $\begin{array}{l}\text { Serum } \beta 2 \text {-microglobulin } \geq \\
1,613 \mathrm{mg} / 1\end{array}$ & $1.83(0.17-20.16)$ & 0.622 & & \\
\hline \multicolumn{5}{|l|}{ Chemotherapyb } \\
\hline MTX & $0.20(0.06-0.70)$ & 0.012 & $\begin{array}{l}0.19 \\
(0.05-0.67)\end{array}$ & 0.010 \\
\hline Rituximab & $0.19(0.04-0.91)$ & 0.037 & & \\
\hline Radiotherapy & $1.65(0.35-7.70)$ & 0.524 & & \\
\hline
\end{tabular}

Age serves as an independent survival predictor due to its association with comorbidity and as an indicator for the possibility of toxic death attributed to chemoradiation [27, 28]. Two large retrospective studies, namely IELSG and MSKCC, showed age as an independent parameter for survival and combined age with other factors to establish models for PCNSL discriminating between different prognostic groups $[15,16]$. Recent studies found that age may confer a time-dependent impact and might play a role in predicting early death. Herve et al. retrospectively evaluated 91 patients with PCNSL, finding that age impacted survival during the initial period but lost its prognostic value after six months [18], which was the approximate time of completion of PCNSL treatment. Our study results are consistent with previous studies, showing age as an independent risk factor for survival, and further identified an age cutoff at 80 conferring clinical relevance for early mortality within 60 days.

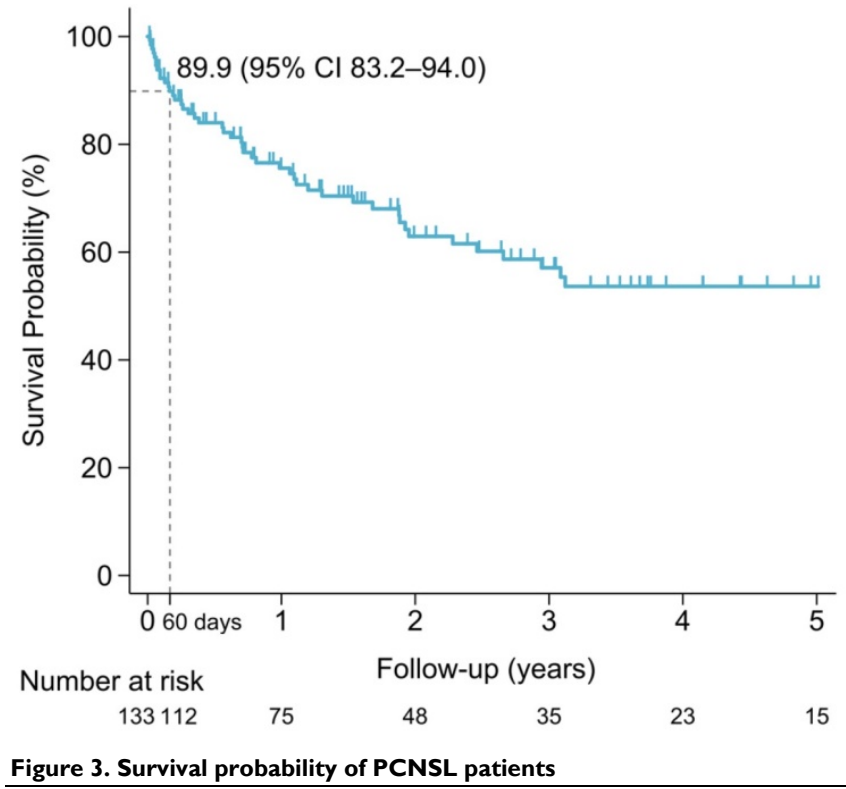

Tumor bleeding, $7.7 \%$

Lymphoma progression, $7.7 \%$

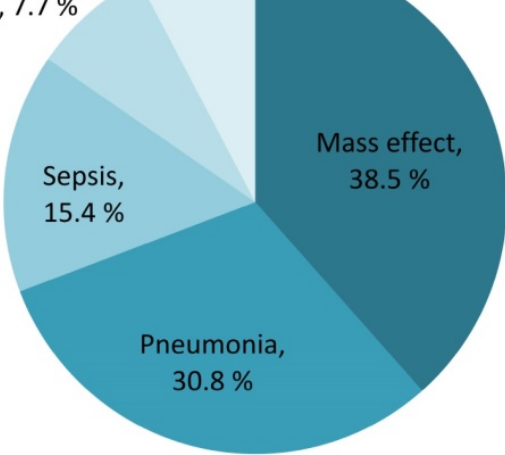

Figure 4. Contributing causes of early mortality (percentage of all mortality)

This study indicates that involvement of the basal ganglia is an independent predictor for early mortality in PCNSL patients. The basal ganglia consist of multiple subcortical regions intertwined with the cerebral cortex, thalamus and brain stem and serves a pivotal role in movement adjustment and initiation. Previously, deep brain involvement, which is defined in IELSG as "including involvement of periventricular regions, the basal ganglia, brainstem or cerebellum," has long been widely reported as an unfavorable prognostic factor for death within six months and for overall survival $[7,15,18]$. In this study, evidence indicated that PCNSL involving specific location such as basal ganglia rather than brain stem or cerebellum was associated with higher risk of early mortality 
within 60 days. Elucidation of the mechanism and external validation for the prognostic value of basal ganglia involvement are definitely required in the future prospective study.

Performance status (PS) has long been accepted as a powerful prognostic factor for PCNSL, which plausibly dictates the treatment plan of patients (doses and chemotherapy regimens). In databases from previous retrospective study (IELSG, MSKCC and Nottingham/Barcelona), PS consistently determined survival of PCNSL patients [15-17]. In our cohort, ECOG more than 1 was associated with early mortality in univariate analysis. Nonetheless, ECOG PS was not a significant risk factor for early mortality in multivariate analysis. This may be explained by the strong correlation between PS and age, of which the effect outweighing that of PS on predicting early mortality. Also, ECOG PS scales have been long criticized for its subjective nature, thus leading to high interobserver variability and recall bias [29].

We further analyzed the potential impact of first-line therapies on early mortality and found that MTX was associated with a significantly reduced risk of early mortality within 60 days. Although no universal consensus has been reached regarding the optimal treatment for PCNSL, MTX-based chemotherapy is the primary building block for the combination of polychemotherapy currently. With the introduction of MTX, prolonged median OS of 30 to 60 months has been observed compared with WBRT alone [3, 30-33]. This study corroborated previous data showing beneficial effect of MTX toward PCNSL patients and further reported MTX as an independent protective factor against early mortality in PCNSL patients.

Immunochemotherapy with rituximab was another potential first-line PCNSL treatment regimens $[3,34]$. In our study, individuals receiving immunochemotherapy with rituximab showed lower risk of early mortality in univariate analysis but did not attain statistical significance in multivariate analysis. As a monoclonal anti-CD20 antibody, rituximab has been shown to confer significant benefits in the treatment of systemic DLBCL.[35] However, the large molecular weight of rituximab limits its penetration across the blood-brain barrier, thus rendering the role of rituximab a long-debated issue in the management of PCNSL.[36, 37] A meta-analysis including six retrospective studies and two phase II randomized controlled trials (RCT) demonstrated that the addition of rituximab was significantly associated with higher complete remission rate, PFS and OS [38]. Nevertheless, the interpretation should be cautious given that one of the included RCT was of poor quality and the heterogeneity among studies was significant with respect to two-year PFS. Additionally, the most recent phase III international RCT $(n=200)$ reported that the incorporation of rituximab into MTX-based regimens did not improve response rates or survival [39]. Herein, we also did not detect significant benefit of rituximab in early mortality of PCSNL after adjustment for other confounding variables, suggesting a marginal role of rituximab regarding early mortality. Certainly, further validation of the contribution of rituximab to early mortality awaits more prospective studies.

CSF protein may reflect tumor burden, leptomeningeal involvement and even disease severity. In the large database form IESLG, CSF protein concentration independently determined survival of PCNSL patients [15]. Yet, several studies later cast doubt on the prognostic value of CSF protein. D'Haene et al. exhibited that the IESLG score without this parameter remains potent in predicting survival independently.[40] Similarly, in another study of 91 PCNSL patients, CSF protein level was not identified as a significant predictive factor in both univariate and multivariate analysis [18]. The present study further demonstrated that increased CSF concentration was not associated with early mortality within 60 days for PCNSL patients. Both our results and previous studies suggest that CSF protein level may not have significant implications for predicting the prognosis of the disease. Surely, more prospective studies are warranted to examine the impact of CSF protein concentration on PCNSL survival prognostication. $\mathrm{LDH}$ serum level is a potential indicator of tissue breakdown and therefore may denote the proliferation rate of tumors. It has been considered an independent factor for predicting OS in PCSNL patients in IELSG [15]. Herve et al. also established the role of serum LDH level in predicting PCNSL survival within six months [18]. However, results from two large cohorts were inconsistent with the aforementioned findings, thereby raising questions in terms of the prognostic value of serum LDH level for survival [16, 41]. In current study, heightened LDH serum level, was also not identified as a risk factor for early mortality within 60 days in either univariate or multivariate analysis. The controversy for the role of serum LDH in predicting OS and early mortality for PCNSL remains and thus requires further study.

In the current study, we reported that around $9.8 \%$ of PCNSL patients suffered from early mortality, with tumor-associated mass effect and infection as two leading causes. Those who succumb to early mortality lose the opportunity of receiving complete cycles of standardized chemotherapy regimens and 
further advanced therapies such as auto-HSCT. Ferreri et al. reported that more than $10 \%$ of patients encountering early mortality before finishing four 21-day cycles of a MTX-based regimen, largely due to acute toxic effect and lymphoma progression.[11] In a phase-3 randomized trial $(n=526)$, Thiel et al. also recorded an estimated 66 patients $(13.0 \%)$ having died during front-line chemotherapy (six 14-day cycles of MTX) before receiving whole brain radiation, majorly related to treatment and disease progression [12]. Knowledge of the main causes and predicting factors for early mortality can help clinicians identify those at-risk patients, take special precautions and even develop modified chemotherapy regimens to extend their survival time. Prior study has investigated the predicting value of IELSG score for early mortality in the earlier cohort (1984-2006) [18]. However, the treatment and survival outcomes for PCNSL patients have improved significantly over the past decades, as indicated by some specialized centers $[10,13,14]$ as well as large national databases $[42,43]$. Therefore, the results by Ghesquières et al. may not be directly generalizable to patients in the contemporary era, thereby highlighting the importance of this research as we investigated patients in more recent periods (2002-2018). When a high-risk population can be identified, prophylactic measures such as prophylactic antimicrobials or approaches for early detection of infections could be prompted and further tested in prospective studies considering infection as a main cause of early mortality.

Our study includes some limitations. The retrospective nature of this study precludes us from drawing solid conclusions from these data. Second, this study spanned an observation period of 16 years, during which the standards of care and diagnosis undoubtedly varied. Furthermore, considering the very small cohort $(n=133)$, it is of difficulty to determine risk factors for early mortality, which could therefore reduce the chance of detecting a true effect. Yet, the extreme rare incidence of PCNSL itself limits the number of patients to be enrolled, making the evidence from the present observational study very important given it is the largest cohort addressing the issue of early mortality of PCNSL to date. Of note, this study may not represent all early-mortality PCNSL patients given that those with suspected PCNSL lesions only in images but without histological confirmation were not included. However, the strict criteria ensure definite diagnosis and prevents the misclassification of patients without PCNSL into this cohort. Finally, the timing of initiating cancer treatment and the choice of which chemotherapy regimens selected as first-line therapy are physician-dependent rather than a standardization of care, and thus it is difficult to evaluate the impact of treatment on early mortality without introducing some unknown confounding factors or bias.

\section{Conclusion}

Despite the development of innovative combination of immunochemotherapies, treating PCNSL remains a challenge. This cohort showed that early mortality occurred in nearly $9.8 \%$ of newly diagnosed PCNSL patients and that infection and tumor-associated mass effect were two leading causes of mortality in these patients. We further identified the risk factors of early mortality, including age $\geq 80$ and basal ganglia involvement. Additionally, individuals receiving MTX-based chemotherapy showed decreased risk of early mortality. Identifying risk factors for early mortality might assist physicians in identification of these patients, as well as the initiation of intensive surveillance and development of prophylactic measures. However, more evidence from prospective studies is needed to further confirm these risk factors.

\section{Abbreviations}

CNS: central nervous system; PCNSL: primary CNS lymphoma; NHL: non-Hodgkin's lymphoma; DLBCL: diffuse large B-cell lymphoma; auto-HSCT: autologous hematopoietic stem cell transplantation; OS: overall survival; PFS: progression-free survival; IELSG: International Extranodal Lymphoma Study Group; MSKCC: Memorial Sloan Kettering Cancer Center; NB: Nottingham-Barcelona; PS: performance status; LDH: lactate dehydrogenase; CSF: cerebrospinal fluid; MRI: magnetic resonance image; CT: computed tomography; WBC: white blood cell; ECOG: the Eastern Cooperative Oncology Group performance score; IQR: interquartile ranges; HRs: hazard ratios; CI: confidence interval; MTX: Methotrexate; WBRT: whole-brain radiation therapy.

\section{Acknowledgements}

This study was supported by grants from Taipei Veterans General Hospital (V105B-016 and V105E10-002-MY2-1), the Ministry of Science and Technology (MOST 104-2314-B-075-085-MY2), the Taiwan Clinical Oncology Research Foundation, Szu-Yuan Research Foundation of Internal Medicine, and Chong Hin Loon Memorial Cancer and Biotherapy Research Center at National Yang-Ming University.

\section{Authors' Contributions}

C-HL and C-JL had full access to all of the data in the study and take responsibility for its integrity as well as the accuracy of the data analysis. C-HL and 
C-JL designed the study. C-FY, C-MY, C-HL, H-YW and C-JL acquired the data and performed statistical analysis. C-FY, T-JC, H-YW, and P-SK provided the final interpretation of the results. C-HL and C-JL drafted the manuscript. Y-CL, C-FY, H-CY, L-YF, J-PG and L-TH made critical revisions to the manuscript for important intellectual content. C-MY, P-MC, and T-JC provided administrative, technical, and material support. C-JL were the study supervisors. C-JL acts as guarantors and accept responsibility for the integrity of the work as a whole. All authors have read and approved the final manuscript.

\section{Competing Interests}

The authors have declared that no competing interest exists.

\section{References}

1. Villano JL, Koshy M, Shaikh H, Dolecek TA, McCarthy BJ. Age, gender, and racial differences in incidence and survival in primary CNS lymphoma. British journal of cancer. 2011; 105: 1414-8.

2. Panageas KS, Elkin EB, DeAngelis LM, Ben-Porat L, Abrey LE. Trends in survival from primary central nervous system lymphoma, 1975-1999: a population-based analysis. Cancer. 2005; 104: 2466-72.

3. Grommes C, DeAngelis LM. Primary CNS Lymphoma. Journal of clinical oncology : official journal of the American Society of Clinical Oncology. 2017; 35: $2410-8$.

4. Hoang-Xuan K, Bessell E, Bromberg J, Hottinger AF, Preusser M, Ruda R, et al. Diagnosis and treatment of primary CNS lymphoma in immunocompetent patients: guidelines from the European Association for Neuro-Oncology. The Lancet Oncology. 2015; 16: e322-32.

5. Swerdlow SH, Campo E, Harris NL, Jaffe ES, Pileri SA, Stein H, et al. WHO Classification of Tumours of Haematopoietic and Lymphoid Tissues. France: IARC. 2008.

6. O'Neill BP, Decker PA, Tieu C, Cerhan JR. The changing incidence of primary central nervous system lymphoma is driven primarily by the changing incidence in young and middle-aged men and differs from time trends in systemic diffuse large B-cell non-Hodgkin's lymphoma. American journal of hematology. 2013; 88: 997-1000.

7. Welch MR, Omuro A, Deangelis LM. Outcomes of the oldest patients with primary CNS lymphoma treated at Memorial Sloan-Kettering Cancer Center. Neuro-oncology. 2012; 14: 1304-11.

8. Henry JM, Heffner RR, Jr., Dillard SH, Earle KM, Davis RL. Primary malignant lymphomas of the central nervous system. Cancer. 1974; 34: 1293-302.

9. Schmitz N. Treatment of primary CNS lymphoma. Blood. 2015; 125: 1360-1.

10. Omuro A, Correa DD, DeAngelis LM, Moskowitz CH, Matasar MJ, Kaley TJ, et al. R-MPV followed by high-dose chemotherapy with TBC and autologous stem-cell transplant for newly diagnosed primary CNS lymphoma. Blood. 2015; 125: 1403-10

11. Ferreri AJ, Reni M, Foppoli M, Martelli M, Pangalis GA, Frezzato M, et al. High-dose cytarabine plus high-dose methotrexate versus high-dose methotrexate alone in patients with primary CNS lymphoma: a randomised phase 2 trial. Lancet (London, England). 2009; 374: 1512-20.

12. Thiel E, Korfel A, Martus P, Kanz L, Griesinger F, Rauch M, et al. High-dose methotrexate with or without whole brain radiotherapy for primary CNS lymphoma (G-PCNSL-SG-1): a phase 3, randomised, non-inferiority trial. The Lancet Oncology. 2010; 11: 1036-47.

13. Rubenstein JL, Hsi ED, Johnson JL, Jung SH, Nakashima MO, Grant B, et al. Intensive chemotherapy and immunotherapy in patients with newly diagnosed primary CNS lymphoma: CALGB 50202 (Alliance 50202). Journal of clinical oncology : official journal of the American Society of Clinical Oncology. 2013; 31: 3061-8.

14. Morris PG, Correa DD, Yahalom J, Raizer JJ, Schiff D, Grant B, et al. Rituximab, methotrexate, procarbazine, and vincristine followed by consolidation reduced-dose whole-brain radiotherapy and cytarabine in newly diagnosed primary CNS lymphoma: final results and long-term outcome. Journal of clinical oncology : official journal of the American Society of Clinical Oncology. 2013; 31: 3971-9.

15. Ferreri AJ, Blay JY, Reni M, Pasini F, Spina M, Ambrosetti A, et al. Prognostic scoring system for primary CNS lymphomas: the International Extranodal Lymphoma Study Group experience. Journal of clinical oncology : official journal of the American Society of Clinical Oncology. 2003; 21: 266-72.

16. Abrey LE, Ben-Porat L, Panageas KS, Yahalom J, Berkey B, Curran W, et al. Primary central nervous system lymphoma: the Memorial Sloan-Kettering
Cancer Center prognostic model. Journal of clinical oncology : official journal of the American Society of Clinical Oncology. 2006; 24: 5711-5.

17. Bessell EM, Graus F, Lopez-Guillermo A, Lewis SA, Villa S, Verger E, et al. Primary non-Hodgkin's lymphoma of the CNS treated with CHOD/BVAM or BVAM chemotherapy before radiotherapy: long-term survival and prognostic factors. International journal of radiation oncology, biology, physics. 2004; 59: $501-8$.

18. Ghesquieres H, Drouet $\mathrm{Y}$, Sunyach MP, Sebban C, Chassagne-Clement C, Jouanneau E, et al. Evidence of time-dependent prognostic factors predicting early death but not long-term outcome in primary CNS lymphoma: a study of 91 patients. Hematological oncology. 2013; 31: 57-64.

19. Dumontet C, Mounier N, Munck JN, Bosly A, Morschauser F, Simon D, et al. Factors predictive of early death in patients receiving high-dose $\mathrm{CHOP}$ (ACVB regimen) for aggressive non-Hodgkin's lymphoma: a GELA study. British journal of haematology. 2002; 118: 210-7.

20. Ray-Coquard I, Ghesquiere H, Bachelot T, Borg C, Biron P, Sebban C, et al. Identification of patients at risk for early death after conventional chemotherapy in solid tumours and lymphomas. British journal of cancer. 2001; 85: 816-22.

21. Oken MM, Creech RH, Tormey DC, Horton J, Davis TE, McFadden ET, et al. Toxicity and response criteria of the Eastern Cooperative Oncology Group. American journal of clinical oncology. 1982; 5: 649-55.

22. Schag CC, Heinrich RL, Ganz PA. Karnofsky performance status revisited: reliability, validity, and guidelines. Journal of clinical oncology : official journal of the American Society of Clinical Oncology. 1984; 2: 187-93.

23. Augustson BM, Begum G, Dunn JA, Barth NJ, Davies F, Morgan G, et al. Early mortality after diagnosis of multiple myeloma: analysis of patients entered onto the United kingdom Medical Research Council trials between 1980 and 2002--Medical Research Council Adult Leukaemia Working Party. Journal of clinical oncology : official journal of the American Society of Clinical Oncology. 2005; 23: 9219-26.

24. Hsu P, Lin TW, Gau JP, Yu YB, Hsiao LT, Tzeng CH, et al. Risk of Early Mortality in Patients With Newly Diagnosed Multiple Myeloma. Medicine. 2015; 94: e2305.

25. Blay JY, Lasset C, Carrie C, Chauvin F, Coiffier B, Gisselbrecht C, et al Multivariate analysis of prognostic factors in patients with non HIV-related primary cerebral lymphoma. A proposal for a prognostic scoring. British journal of cancer. 1993; 67: 1136-41.

26. Pollack IF, Lunsford LD, Flickinger JC, Dameshek HL. Prognostic factors in the diagnosis and treatment of primary central nervous system lymphoma. Cancer. 1989; 63: 939-47.

27. Reni M, Ferreri AJ, Garancini MP, Villa E. Therapeutic management of primary central nervous system lymphoma in immunocompetent patients: results of a critical review of the literature. Annals of oncology : official journal of the European Society for Medical Oncology / ESMO. 1997; 8: 227-34.

28. Hayabuchi N, Shibamoto Y, Onizuka Y. Primary central nervous system lymphoma in Japan: a nationwide survey. International journal of radiation oncology, biology, physics. 1999; 44: 265-72.

29. Kelly CM, Shahrokni A. Moving beyond Karnofsky and ECOG Performance Status Assessments with New Technologies. Journal of oncology. 2016; 2016: 6186543.

30. Poortmans PM, Kluin-Nelemans HC, Haaxma-Reiche $H$, Van't Veer $M$ Hansen M, Soubeyran P, et al. High-dose methotrexate-based chemotherapy followed by consolidating radiotherapy in non-AIDS-related primary central nervous system lymphoma: European Organization for Research and Treatment of Cancer Lymphoma Group Phase II Trial 20962. Journal of clinical oncology : official journal of the American Society of Clinical Oncology. 2003; 21: 4483-8.

31. Glass J, Gruber ML, Cher L, Hochberg FH. Preirradiation methotrexate chemotherapy of primary central nervous system lymphoma: long-term outcome. J Neurosurg. 1994; 81: 188-95.

32. Abrey LE, Yahalom J, DeAngelis LM. Treatment for primary CNS lymphoma: the next step. Journal of clinical oncology : official journal of the American Society of Clinical Oncology. 2000; 18: 3144-50.

33. O'Brien P, Roos D, Pratt G, Liew K, Barton M, Poulsen M, et al. Phase II multicenter study of brief single-agent methotrexate followed by irradiation in primary CNS lymphoma. Journal of clinical oncology : official journal of the American Society of Clinical Oncology. 2000; 18: 519-26.

34. Fritsch $\mathrm{K}$, Kasenda B, Hader $\mathrm{C}$, Nikkhah G, Prinz M, Haug V, et al Immunochemotherapy with rituximab, methotrexate, procarbazine, and lomustine for primary CNS lymphoma (PCNSL) in the elderly. Annals of oncology : official journal of the European Society for Medical Oncology / ESMO. 2011; 22: 2080-5.

35. Coiffier B, Lepage E, Briere J, Herbrecht R, Tilly H, Bouabdallah R, et al. CHOP chemotherapy plus rituximab compared with $\mathrm{CHOP}$ alone in elderly patients with diffuse large-B-cell lymphoma. The New England journal of medicine. 2002; 346: 235-42.

36. Rubenstein JL, Combs D, Rosenberg J, Levy A, McDermott M, Damon L, et al. Rituximab therapy for CNS lymphomas: targeting the leptomeningeal compartment. Blood. 2003; 101: 466-8.

37. Ruhstaller TW, Amsler U, Cerny T. Rituximab: active treatment of central nervous system involvement by non-Hodgkin's lymphoma? Annals of oncology : official journal of the European Society for Medical Oncology. 2000; 11: $374-5$. 
38. Song $Y$, Wen $Y$, Xue W, Zhang $Y$, Zhang M. Effect of rituximab on primary central nervous system lymphoma: a meta-analysis. International journal of hematology. 2017; 106: 612-21.

39. Bromberg JEC, Issa S, Bakunina K, Minnema MC, Seute T, Cull G, et al. OS2.5 Effect of rituximab in primary central nervous system lymphoma - results of the randomized phase III HOVON 105/ALLG NHL 24 study. Neuro-Oncology. 2018; 20: iii220-iii.

40. D'Haene N, Catteau X, Maris C, Martin B, Salmon I, Decaestecker C. Endothelial hyperplasia and endothelial galectin-3 expression are prognostic factors in primary central nervous system lymphomas. British journal of haematology. 2008; 140: 402-10.

41. Ahn Y, Ahn HJ, Yoon DH, Hong JY, Yoo C, Kim S, et al. Primary central nervous system lymphoma: a new prognostic model for patients with diffuse large B-cell histology. Blood research. 2017; 52: 285-92.

42. Shiels MS, Pfeiffer RM, Besson C, Clarke CA, Morton LM, Nogueira L, et al. Trends in primary central nervous system lymphoma incidence and survival in the U.S. British journal of haematology. 2016; 174: 417-24.

43. Mendez JS, Ostrom QT, Gittleman H, Kruchko C, DeAngelis LM, Barnholtz-Sloan JS, et al. The elderly left behind-changes in survival trends of primary central nervous system lymphoma over the past 4 decades. Neuro Oncol. 2018; 20: 687-94. 